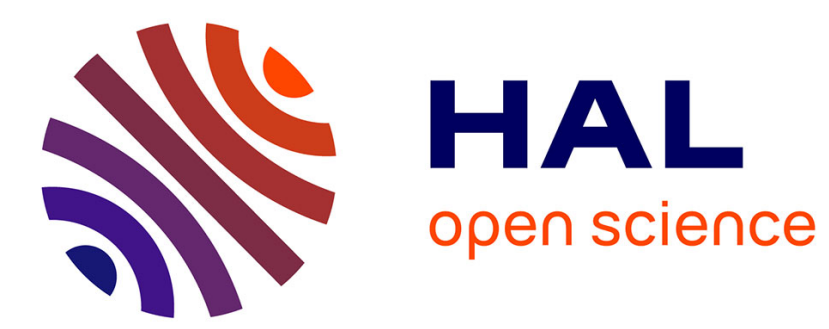

\title{
Multi-traçage de la Fontaine de Nîmes
}

Jean-Christophe Maréchal, Nathalie Courtois, Gilbert Jouanen, Philippe Meus

\section{To cite this version:}

Jean-Christophe Maréchal, Nathalie Courtois, Gilbert Jouanen, Philippe Meus. Multi-traçage de la Fontaine de Nîmes. KARSTOLOGIA, 2010, 56, pp.1-8. hal-00662976

\section{HAL Id: hal-00662976 https://hal.science/hal-00662976}

Submitted on 25 Jan 2012

HAL is a multi-disciplinary open access archive for the deposit and dissemination of scientific research documents, whether they are published or not. The documents may come from teaching and research institutions in France or abroad, or from public or private research centers.
L'archive ouverte pluridisciplinaire HAL, est destinée au dépôt et à la diffusion de documents scientifiques de niveau recherche, publiés ou non, émanant des établissements d'enseignement et de recherche français ou étrangers, des laboratoires publics ou privés. 


\title{
MULTI-TRACAGE DE LA FONTAINE DE NIMES
}

\section{Multi-tracing of Fontaine de Nîmes}

\author{
Jean-Christophe Maréchal ${ }^{1}$, Nathalie Courtois ${ }^{1}$, Gilbert Jouanen ${ }^{2}$, Philippe Meus ${ }^{3}$ \\ ${ }^{1}$ Bureau de Recherches Géologiques et Minières (BRGM) - Service Eau, Unité NRE, 1039 rue de Pinville, 34000 Montpellier, \\ France \\ Tél: +33 (0)4 671579 65, Fax: +33 (0)4 676458 51, e-mail: jc.marechal@brgm.fr \\ ${ }^{2}$ Association Fontaine de Nîmes, e-mail : g.jouanen@free.fr \\ ${ }^{3}$ European Water Tracing Services, rue de la Chapelle 43, B-4550 Nandrin, Belgique, e-mail : ewts@skynet.be
}

\section{RESUME}

Le système karstique des garrigues nîmoises possède un exutoire principal, la Fontaine de Nîmes. Par le passé, plusieurs essais de traçages ont permis de mettre en évidence les relations entre des points supposés de son bassin d'alimentation et la Fontaine de Nîmes. Ces essais manquaient cependant de courbes de restitution. Dans cet article, les résultats d'un multi-traçage effectué en mars 2006 à partir de quatre points d'injection sont présentés. Le réseau de surveillance de cet essai a été conçu de façon à caractériser les rôles respectifs des différents conduits karstiques reconnus menant à la source principale. Les résultats majeurs sont les suivants : (i) tous les traceurs ont été identifiés à la Fontaine de Nîmes (ii) le conduit karstique ouest joue un rôle majeur dans le drainage du bassin d'alimentation de la Fontaine (iii) aucun traceur n'est identifié sur le conduit karstique nord, laissant présager un rôle mineur dans le drainage des parties ouest et nord du bassin d'alimentation (iv) un débit très faible, mesuré par dilution, transite dans la Galerie des Poteries dont le bassin d'alimentation est géographiquement très limité. Par ailleurs, des courbes de restitution bien structurées ont été observées pour trois des quatre essais.

\begin{abstract}
The main outlet of the Nimes karst system is the Fontaine de Nimes spring. In the past, several tracing experiments have identified relationships between points supposed to be part of the recharge area of the system and the Fontaine de Nimes spring. Nevertheless, these tracing experiments suffered from a lack of breakthrough curves. In this paper, the results of a multi-tracing test realized in March 2006 from four injection points are described. The monitoring network of this experiment has been designed in order to characterize the respective roles of the three karst conduits (west karst conduit, north karst conduit and Galerie des Poteries) leading to the main spring. The major results are following: (i) all the dye tracers have been identified at the Fontaine de Nimes spring (ii) the west karst conduit plays a major role in the drainage of the recharge area of the karst system (iii) no dye tracer has been identified on the north karst conduit (iv) the discharge rate into the Galerie des Poteries, measured by dilution of salt upstream injected, is less than 50 $1 / \mathrm{min}$, the recharge area of this conduit is geographically very limited. We can notice that well-structured breakthrough curves have been obtained for three of the four tracers.
\end{abstract}

\section{MOTS CLEFS}

Traçage, source karstique, hydrogéologie, restitution, jaugeage, conduit karstique, réseau spéléologique, Nîmes

\section{KEYWORDS}

Tracing, karst spring, hydrogeology, breakthrough curve, rating curve, karst conduit, speleological network, Nimes 


\section{INTRODUCTION}

Le système karstique de la Fontaine de Nîmes (Fabre et Guyot 1988) a fait l'objet de plusieurs essais de traçage dans le passé, de façon essentiellement à préciser l'extension de son bassin d'alimentation (Fabre, 1997). Les résultats de ces traçages ont permis d'établir un certain nombre de relations avérées entre les points d'injection et la Fontaine de Nîmes. Cependant, il s'agissait dans tous les cas de traçages que l'on peut qualifier de «qualitatifs », s'arrêtant à l'identification d'une relation sans la quantifier au moyen de courbes de restitution.

L'objectif de cet article est de présenter les résultats d'un premier multi-traçage réalisé en mars 2006 sur la Fontaine de Nîmes avec un suivi quantitatif de l'arrivée des traceurs. En complément, le réseau d'observation a été conçu de façon à caractériser les rôles respectifs des différents drains karstiques alimentant l'exutoire principal du système, et identifiés par les spéléologues (Coste et Jouanen 2006).

\section{ZONE D'ETUDE}

Le système karstique de la Fontaine de Nîmes est un karst gravitaire unaire (Maréchal et al. 2008). Son bassin d'alimentation (Maréchal et al. 2005) se situe dans les garrigues nîmoises constituées de terrains calcaires et marneux du Crétacé (Figure 1).

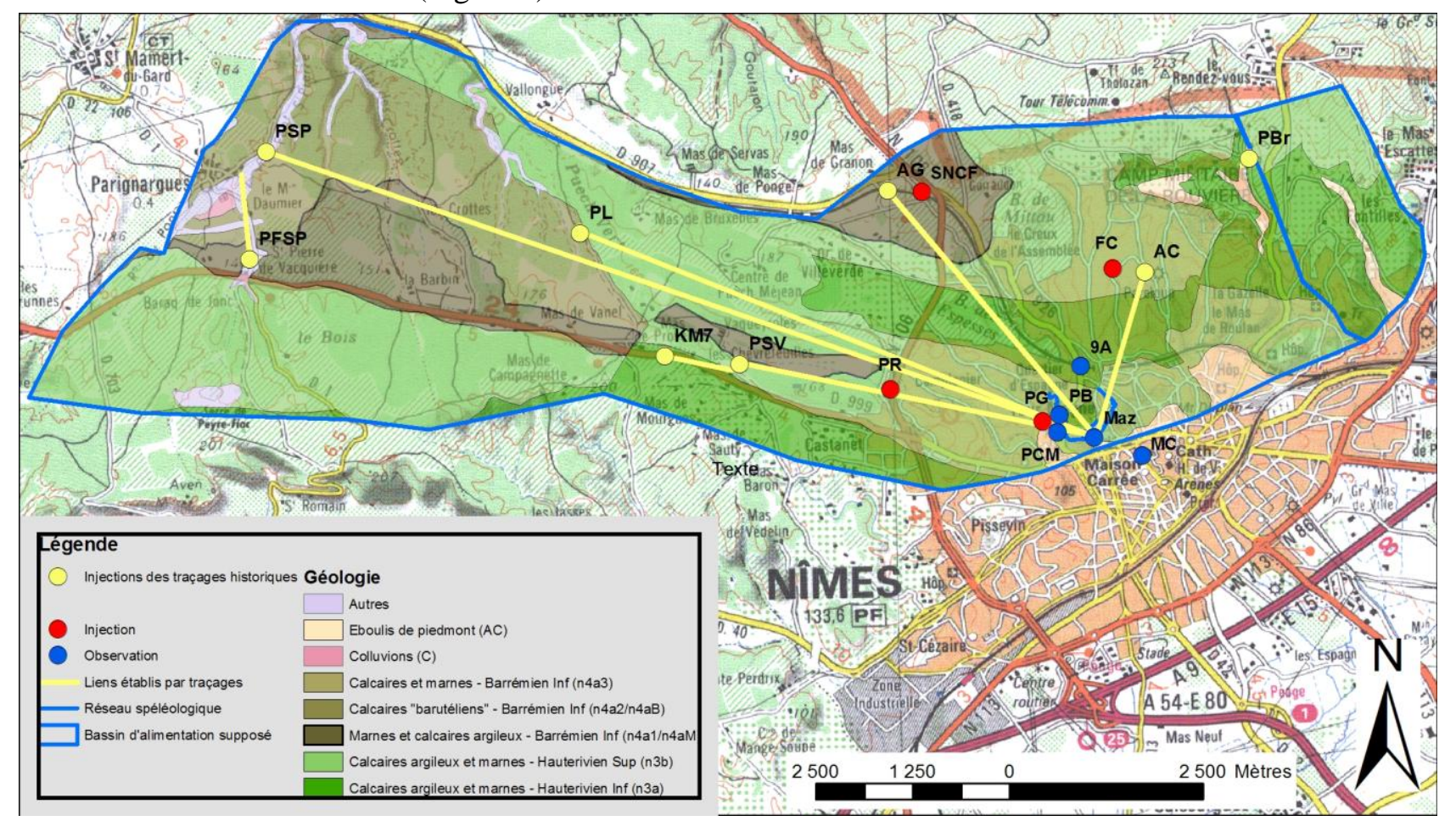

Figure 1 : localisation du système karstique de la Fontaine de Nîmes, de son bassin d'alimentation présumé et de la Ville de Nîmes. Localisation des points d'injection (points jaunes) et des connexions établies lors des traçages précédents (lignes jaunes). Localisation des points d'injection (points rouges) et points de mesures (points bleus) du multi-traçage de mars 2006.

(KM7 : Aven du km 7 ; PSV : Perte de la S. Vaqueyroles ; PFSP : Perte de la Font de St-Pierre ; PSP : Perte de la S. Parignargues ; PL : Perte des Lauzières ; SNCF : Aven du pont SNCF ; FC : Aven de Font Chapelle ; PR :

Aven du pont de la République ; PG : Puits de la Gaffone ; 9A : Puits des 9 Arcades ; PB : Puits Poubelle ; PCM : Puits du Creux de Mouleri ; Maz : Fontaine de Nîmes à l'Aven Mazauric ; MC : Maison Carrée ; AC : Aven du Chenal)

Figure 1: location of Fontaine de Nimes karst system, supposed recharge area and city of Nimes. Location of injection points (yellow dots) and relationships established during previous tracing experiments (yellow lines). Location of injection points (red dots) and observation points (blue dots) during the multi-tracing experiment of March 2006.

Les exutoires du système sont la source Fontaine de Nîmes ( $\mathrm{FdN}$ ), avec un débit compris entre 0,01 et 30 $\mathrm{m}^{3} / \mathrm{s}$ (maximum estimé pour l'épisode de 1988), et plusieurs sources temporaires fonctionnant seulement durant les crues éclair (Maréchal et Ladouche 2006 ; Maréchal et al. 2008 ; Maréchal et al. 2009). Le débit moyen de la FdN est estimé à $0,55 \mathrm{~m}^{3} / \mathrm{s}$ sur la période 1998-2005 (Maréchal et al. 2006). Le système 
karstique est le siège d'écoulements hiérarchisés vers la source de la Fontaine de Nîmes (FdN) au travers de plusieurs drains karstiques (Figure 2) cartographiés par les spéléologues de l'Association Fontaine de Nîmes (Coste et Jouanen 2006), et listés ci-dessous par ordre décroissant d'importance : (i) le conduit Ouest, (ii) le conduit Nord et (iii) la Galerie des Poteries explorée et topographiée en 1991 (Coste et al. 1991 ; Coste et Jouanen 2003).

Un certain nombre d'essais de traçage que l'on peut qualifier de «qualitatifs » dans la mesure où aucune courbe de restitution n'est disponible, ont été précédemment réalisés dans le secteur de la Fontaine de Nîmes (Tableau 1). On y ajoutera le traçage effectué entre l'Aven du Chenal (AC) et la Fontaine de de Nîmes.

\begin{tabular}{|c|c|c|c|c|c|c|c|c|c|c|c|c|}
\hline Lieu injection & Id & Auteur & Date & $\begin{array}{c}\boldsymbol{M} \\
(\mathbf{k g})\end{array}$ & $\begin{array}{c}\boldsymbol{Q}_{i} \\
(\mathbf{l} / \mathbf{s})\end{array}$ & $\begin{array}{c}\text { Lieu } \\
\text { réapparition }\end{array}$ & $\begin{array}{c}\boldsymbol{Q}_{r} \\
(\mathbf{l} / \mathbf{s})\end{array}$ & $\begin{array}{c}\boldsymbol{T} \\
(\mathbf{j})\end{array}$ & $\begin{array}{c}\boldsymbol{V} \\
(\mathbf{m} / \mathbf{h})\end{array}$ & $\begin{array}{c}\boldsymbol{D} \\
(\mathbf{k m})\end{array}$ & $\begin{array}{c}\boldsymbol{H} \\
(\mathbf{m})\end{array}$ & $\begin{array}{c}\boldsymbol{P} \\
(\boldsymbol{\%})\end{array}$ \\
\hline Aven du km 7 & KM7 & A. Bonnet & $9 / 1950$ & & 0,3 & Vaqueyroles & & 2 & 18 & 0,8 & 5 & 0,6 \\
\hline $\begin{array}{c}\text { Perte de la S. } \\
\text { Vaqueyroles }\end{array}$ & PSV & $\begin{array}{c}\text { ASN- } \\
\text { BRGM }\end{array}$ & $30 / 11 / 1966$ & 5 & 0,1 & F. de Nîmes & 100 & 26 & 7,6 & 4,7 & 74 & 1,6 \\
\hline $\begin{array}{c}\text { Perte de la Font } \\
\text { de St-Pierre }\end{array}$ & PFSP & BRGM & $8 / 12 / 1967$ & 5 & 2 & F. de Nîmes & & 31 & 1,2 & 11 & 8 & 0,9 \\
\hline $\begin{array}{c}\text { Perte de la S. } \\
\text { Parignargues }\end{array}$ & PSP & & & & & F. de Nîmes & & 16 & & 11 & 73 & 0,6 \\
\hline $\begin{array}{c}\text { Perte des } \\
\text { Lauzières }\end{array}$ & PL & G. Fabre & $18 / 5 / 1978$ & 10 & 112 & F. de Nîmes & 750 & 3,5 & 92 & 7,8 & 91 & 1,2 \\
\hline
\end{tabular}

Tableau $1:$ synthèse des essais de traçage réalisés dans le secteur de la Fontaine de Nîmes ( $M:$ masse de fluorescéine injectée ; $Q i$ : débit à l'injection; $Q r$ : débit à la réapparition; $T$ : temps de passage ; $V$ : vitesse théorique ; $D$ : distance $; H$ : dénivelé ; $P$ : pente théorique)

Table 1: summary of previous tracing experiments in Fontaine de Nîmes area (M: injected tracer mass; $Q_{i}:$ discharge rate at injection; $Q_{r}$ : discharge rate at observation point; $T$ : tracer recovery time; $V$ : theoretical velocity; $D$ : distance; H: elevation variation; $P$ : theoretical slope)

\section{MULTI-TRAÇAGE DE MARS 2006}

\section{III.1 Points d'injection}

Le multi-traçage de la Fontaine de Nîmes a été réalisé le 10 mars 2006. Trois traceurs fluorescents (uranine, sulforhodamine $\mathrm{G}$ et naphtionate de sodium) ainsi qu'un traceur salin $(\mathrm{NaCl})$ ont été injectés le même jour à quatre endroits différents. Chacune des injections de traceur a été suivie d'une chasse de plusieurs $\mathrm{m}^{3}$ d'eau avec l'aide des pompiers de la Ville de Nîmes. Les caractéristiques des injections sont résumées au Tableau 2. Les quantités injectées ont été déterminées de façon à réduire les masses de traceurs tout en s'assurant d'obtenir, à la Fontaine de Nîmes, des concentrations supérieures aux seuils de détection des méthodes d'analyses.

\begin{tabular}{|c|c|c|c|c|c|c|}
\hline Lieu & Identifiant & $\begin{array}{l}\text { Distance à } \\
\text { FdN (m) }\end{array}$ & Heure & Traceur & $\begin{array}{c}\text { Quantité } \\
\text { (kg) }\end{array}$ & $\begin{array}{c}\text { Eau ajoutée } \\
\left(\mathbf{m}^{3}\right)\end{array}$ \\
\hline Aven du pont SNCF & SNCF & 3900 & $09: 25$ & uranine & 2 & 10 \\
\hline Aven de Font Chapelle & FC & 2000 & $10: 38$ & sulforhodamine $\mathrm{G}$ & 3 & 10 \\
\hline $\begin{array}{l}\text { Aven du pont de la } \\
\text { République }\end{array}$ & PR & 2700 & $13: 45$ & $\begin{array}{l}\text { naphtionate de } \\
\text { sodium }\end{array}$ & 12,5 & 10 \\
\hline Puits de la Gaffone & PG & 700 & $15: 30$ & sel $(\mathrm{NaCl})$ & 15 & 6 \\
\hline
\end{tabular}

Tableau 2 : synthèse des points d'injection

Table 2 : summary of injection points

L'aven du Pont SNCF n'avait jamais été tracé. Situé à près de $3 \mathrm{~km}$ au Nord-Ouest de la Fontaine, il était intéressant de confirmer son appartenance au bassin d'alimentation de la Fontaine. Les injections aux avens du Pont de la République et de Font Chapelle étaient destinées à identifier les rôles respectifs des conduits karstiques Ouest et Nord. L'injection au Puits de la Gaffone relevait plutôt de l'intention de jauger le débit de la Galerie des Poteries entre le point d'injection et le Creux de Mouleri (Figure 2). 
L'injection au Pont de la République permettait également d'évaluer le rôle du vallon de Vaqueyroles qui se trouve légèrement au N-O de la ville, considéré par certains auteurs (Nejad Hachemi, 1968) comme une zone d'alimentation principale de la Fontaine de Nîmes. Il est formé par un synclinal des calcaires compacts de l'Hauterivien supérieur dont le cour est constitué par un lambeau de calcaire très marneux du Barrémien inférieur. Selon Nejad Hachemi (1968), le substratum marno-calcaire ayant constitué le niveau de base karstique, les eaux infiltrées ont développé un réseau souterrain qui suit l'axe ONO- ESE du synclinal incliné vers Nîmes et qui débouche au travers de la branche Ouest de la Fontaine située dans le prolongement de cet axe.

Lors de l'injection le 10 mars 2006, soit plus d'un mois après l'épisode de crue de fin janvier 2006 (débit maximal de la Fontaine de l'ordre de $6,4 \mathrm{~m}^{3} / \mathrm{s}$ ), le débit à la Fontaine est en phase de récession. Il est de l'ordre de $0,46 \mathrm{~m}^{3} / \mathrm{s}$, un peu inférieur au débit moyen $\left(Q_{\text {moy }}=0,55 \mathrm{~m}^{3} / \mathrm{s}\right)$. Entre le 10 mars et la fin juin 2006 , durant la restitution des traceurs, on n'enregistre aucun épisode de pluie significatif et le tarissement se poursuit. On peut donc qualifier l'état hydrologique du système de «moyennes à basses eaux » et «non influencé par l'infiltration ».

\section{III.2 Réseau d'observation}

Parallèlement à la détermination des relations potentielles à longue distance, nous avons souhaité investiguer le rôle des différents conduits karstiques alimentant l'exutoire principal du système, la Fontaine de Nîmes. 


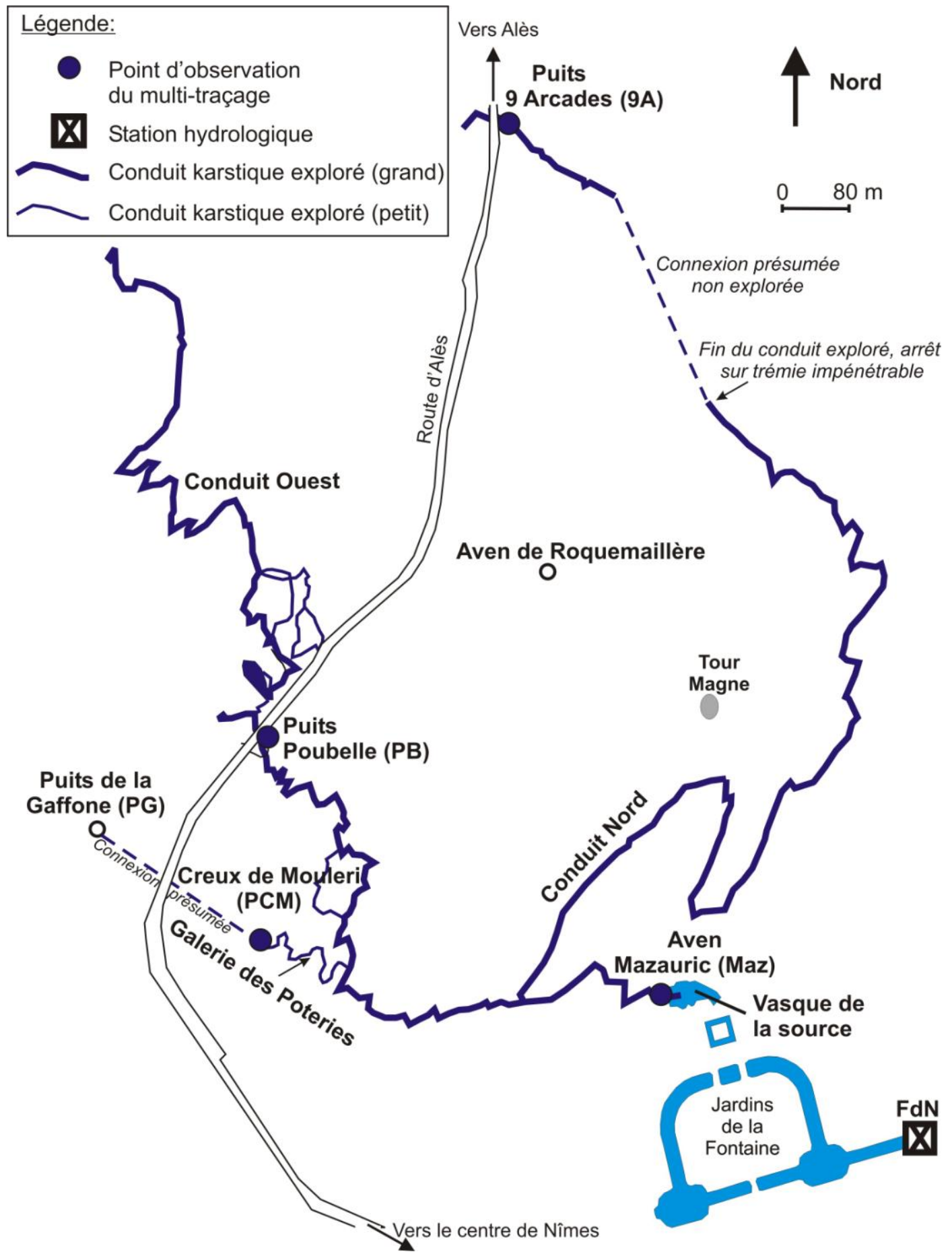

Figure 2 : réseau spéléologique de la Fontaine de Nîmes et localisation du réseau d'observation (modifié d'après l'Association Fontaine de Nîmes)

Figure2: speleological network of Fontaine de Nimes and location of observation network (modified after Association Fontaine de Nimes)

Cinq points de suivi ont été définis afin d'optimiser le réseau de surveillance (Figures 1 et 2):

- Puits des 9 Arcades (9A) sur le conduit Nord;

- Puits Poubelle (PB) sur le conduit Ouest;

- Puits du Creux de Mouleri (PCM) sur la Galerie des Poteries ;

- Fontaine de Nîmes à l'Aven Mazauric (Maz) situé quelques mètres à l'ouest et en relation directe avec la vasque de la Fontaine;

- Maison Carrée (MC) dans les dépôts quaternaires à l'aval (Figure 1). 


\section{III.3 Suivi et analyses}

Le suivi des restitutions sur ces différents points a été effectué en combinant suivi en continu au moyen d'équipements en place, et suivi manuel. Le récapitulatif des modalités de suivi est donné dans le Tableau 3.

\begin{tabular}{|c|c|c|c|c|c|c|c|}
\hline & \multicolumn{3}{|c|}{ Equipement } & \multicolumn{3}{|c|}{ Suivi manuel } & \multirow[b]{2}{*}{$\begin{array}{l}\text { Date de fin } \\
\text { de suivi }\end{array}$} \\
\hline Point suivi & $\begin{array}{c}\text { Fluorimètre de terrain } \\
\text { (uranine et } \\
\text { amidorhodamine G) }\end{array}$ & $\begin{array}{c}\begin{array}{c}\text { Sonde de } \\
\text { conductivité } \\
(\mathrm{NaCl})\end{array} \\
\end{array}$ & $\begin{array}{l}\text { Préleveur } \\
\text { automatique }\end{array}$ & $\begin{array}{c}\text { Echantillon } \\
\text { manuel }\end{array}$ & $\begin{array}{l}\text { Fluocapteurs } \\
\text { charbon actif }\end{array}$ & $\begin{array}{l}\text { Opérateurs pour les } \\
\text { échantillons manuels } \\
\text { et les fluocapteurs }\end{array}$ & \\
\hline $\begin{array}{c}\text { Puits des } 9 \text { Arcades } \\
\text { (Mme Robert) }\end{array}$ & $1 \mathrm{mes} . / 4 \mathrm{~min}$ & & & $1 /$ jour & $1 /$ tournée & Mme Robert / Brgm & $18 / 04 / 2006$ \\
\hline $\begin{array}{l}\text { Puits Poubelle } \\
\left(n^{\circ} 6, \text { puis } n^{\circ} 2\right)\end{array}$ & & & & $\begin{array}{c}1 \text { / jour puis } 1 \text { tous } \\
\text { les } 2-3 \text { jours }\end{array}$ & $\begin{array}{c}1 \text { / jour puis } 1 \text { tous } \\
\text { les } 2-3 \text { jours }\end{array}$ & Spéléologues & $23 / 06 / 2006$ \\
\hline $\begin{array}{c}\text { Puits Pesenti (Creux } \\
\text { de Mouléry) }\end{array}$ & $1 \mathrm{mes} . / 15 \mathrm{~min}$ & $1 \mathrm{mes} / \mathrm{5} \mathrm{min}$ & & $1 /$ jour & $\begin{array}{c}1 \text { / jour puis } 1 \text { tous } \\
\text { les } 2 \text { jours }\end{array}$ & Spéléologues & $23 / 06 / 2006$ \\
\hline Maison Carrée & & & & 1 / tournée & 1 / tournée & Brgm & $18 / 04 / 2006$ \\
\hline Fontaine de Nîmes & & $1 \mathrm{mes} . / 15 \mathrm{~min}$ & 1 éch. $/ 4 \mathrm{~h}$ à $12 \mathrm{~h}$ & $1 /$ jour & $1 /$ jour & Spéléologues / Brgm & $23 / 06 / 2006$ \\
\hline
\end{tabular}

Tableau 3 : Récapitulatif des points de surveillance et des modalités de suivi des restitutions

Table 3: summary of monitoring network and equipment

Pour le suivi du traçage au sel au Puits de la Gaffone, nous avons installé deux sondes de conductivité, avec centrale d'acquisition Logosens, sur le Puits du Creux du Moulery (pas d'enregistrement : 5 min) et la Fontaine de Nîmes (pas d'enregistrement : 15 min).

Pour le suivi des restitutions des traceurs fluorescents, nous avons installé deux fluorimètres de terrain GGUN-FL10 (modèle en forage) au Puits des 9 Arcades (pas d'acquisition : 4 min), et au Puits du Creux du Moulery (pas d'acquisition : $15 \mathrm{~min}$ ). Dans le cas présent, les appareils étaient calibrés pour la détection de l'uranine et de la sulforhodamine $\mathrm{G}$, avec un seuil de détection inférieur à $0,05 \mathrm{ppb}(1 \mathrm{ppb}=1 \mu \mathrm{g} / \mathrm{l})$.

Enfin à la Fontaine de Nîmes, nous avons installé un préleveur automatique autonome de 24 flacons en polyéthylène, alimenté par batterie de $12 \mathrm{~V}$. Les échantillons étaient relevés par le BRGM lors de ses tournées, avec transvasement des échantillons dans des flacons de $250 \mathrm{ml}$ en polyéthylène ambré, pour les garantir de la lumière. Les pas de temps des prélèvements ont varié entre 4 et 12 heures, en fonction des résultats des restitutions.

Les échantillons ont ensuite été envoyés au laboratoire European Water Tracing Services (EWTS), pour analyse des trois traceurs (uranine, sulforhodamine G, et naphtionate de sodium) par spectrofluorimétrie au moyen d'un spectrophotomètre de fluorescence HITACHI F-2500. Les analyses ont été réalisées aux longueurs d'ondes optimales d'excitation et d'émission des trois traceurs, avec confirmation de l'identification de ces traceurs au moyen de spectres par balayage synchronisé.

Ces suivis en continu ont été complétés (et sécurisés) par des suivis manuels, avec la collaboration des spéléologues de l'Association Fontaine de Nîmes, ainsi que des propriétaires du Puits des 9 Arcades, que nous remercions ici vivement pour leur aide précieuse.

Le suivi manuel a concerné : (i) le prélèvement d'échantillons d'eau, dans des flacons en polyéthylène ambré; (ii) l'installation et la récupération de fluocapteurs au charbon actif. Après récupération, ces fluocapteurs ont été stockés dans des sacs étanches aux UV.

Les fluocapteurs au charbon actif ont été utilisés pour doubler (et sécuriser) les informations quantitatives apportées par les fluorimètres de terrain et les analyses d'eau en laboratoire. Aussi, ils n'ont pas été analysés de façon systématique, mais seulement dans certains cas où pouvait subsister un doute quant à la restitution. L'analyse des fluocapteurs a également eu lieu par spectrofluorimétrie, par balayage synchronisé, sur les extraits obtenus à partir d'une solution alcoolique (éthanol) de potasse.Résultats

\section{III.4 Traçage 1 depuis l'aven du Pont SNCF}

Les courbes de restitution de l'uranine ne sont pas bien structurées. Cependant, l'arrivée du traceur est avérée au Puits Poubelle (PB) et à la Fontaine de Nîmes (Maz).

La restitution de l'uranine au Puits Poubelle F2 est demeurée à des niveaux de concentrations très bas (inférieurs à $1 \mu \mathrm{g} / \mathrm{l}$ ). Elle est cependant attestée par les spectres par balayage qui la différencient bien de la fluorescence «naturelle ». Les premières traces sont décelables plus d'un mois après l'injection $(35,4$ jours), ce qui correspond à une vitesse maximale de transit de l'ordre de $4 \mathrm{~m} / \mathrm{h}$ (Tableau 4 ). 
La restitution de l'uranine à la Fontaine de Nîmes est également demeurée toujours à des niveaux de concentrations assez bas, avec un maximum enregistré de 2,3 $\mu \mathrm{g} / \mathrm{l}$. Les premières traces apparaissent plus d'un mois et demi après l'injection (49 jours), ce qui correspond à une vitesse de l'ordre de $3,3 \mathrm{~m} / \mathrm{h}$. Le temps moyen d'arrivée (correspondant à une moyenne pondérée des arrivées) est de 61 jours, ce qui correspond à une vitesse moyenne de $2,7 \mathrm{~m} / \mathrm{h}$. Lors de la dernière mesure le 23 juin 2006, la masse cumulée récupérée est de l'ordre de $330 \mathrm{~g}$, soit $17 \%$ environ de la masse injectée. Ce taux de restitution reste cependant très incertain en raison du faible taux d'échantillonnage au-delà de soixante jours après l'injection.

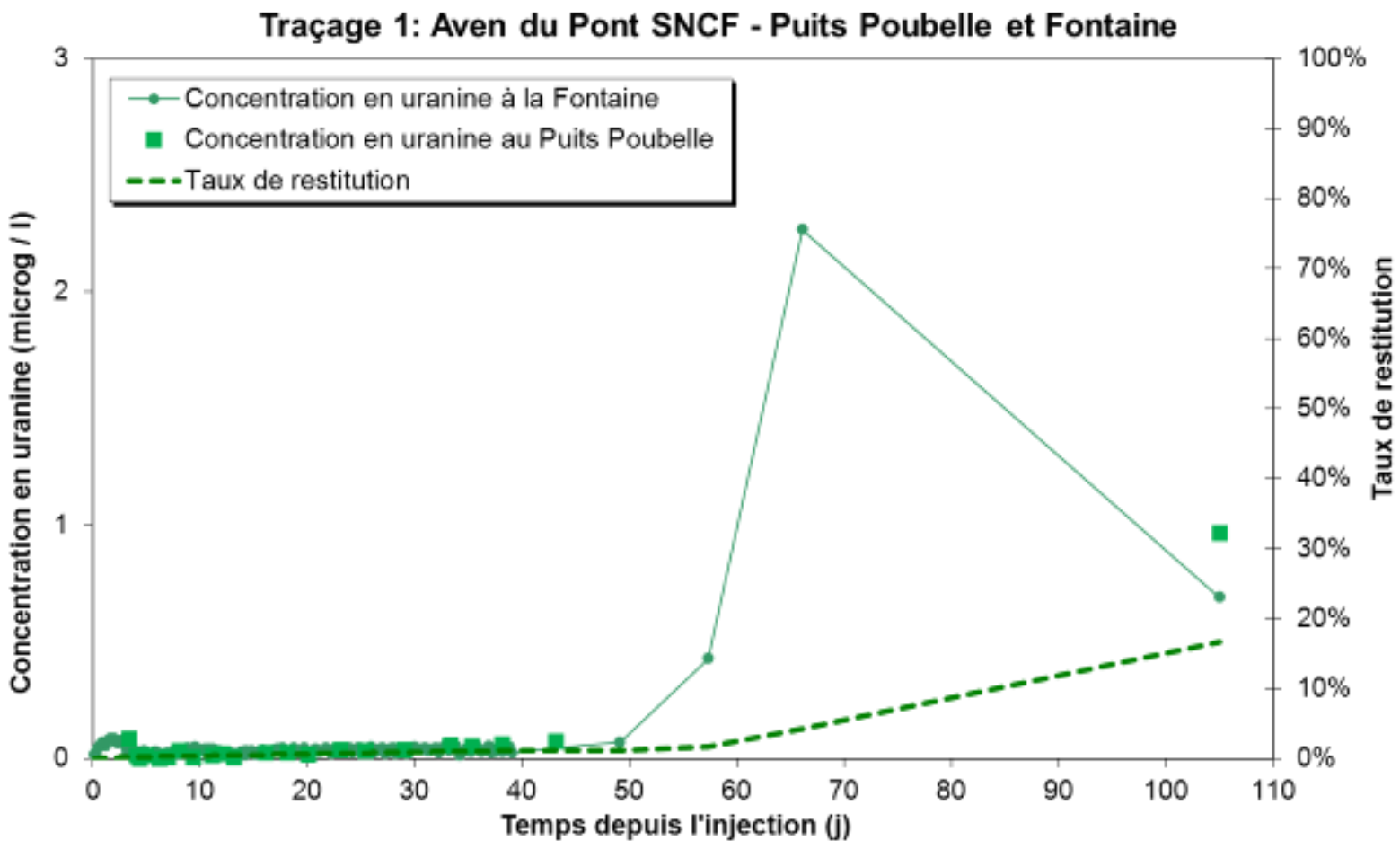

Figure 3 : courbes de restitution d'uranine au puits Poubelle et à la Fontaine de Nîmes

Figure 3: uranin dye breakthrough curves at Poubelle well and Fontaine de Nimes

\begin{tabular}{|c|c|c|c|}
\hline \multicolumn{2}{|c|}{ Restitution des $2 \mathrm{~kg}$ d'uranine } & $\begin{array}{c}\text { Puits Poubelle } \\
\text { (PB) }\end{array}$ & Fontaine de Nîmes \\
\hline & Distance (m) & 3400 & 3900 \\
\hline \multirow{4}{*}{$\begin{array}{c}\text { Première arrivée du } \\
\text { traceur }\end{array}$} & Date & 14-avr-06 & 28-avr-06 \\
\hline & $\begin{array}{l}\text { Temps de transit } \\
\text { minimum }(\mathrm{j})\end{array}$ & 35,4 & 49,1 \\
\hline & $\begin{array}{l}\text { Vitesse maximale de } \\
\text { transit }(\mathrm{m} / \mathrm{j})\end{array}$ & 96 & 79 \\
\hline & $\begin{array}{l}\text { Vitesse maximale de } \\
\text { transit }(\mathrm{m} / \mathrm{h})\end{array}$ & 4,0 & 3,3 \\
\hline \multirow{5}{*}{ Mode de la restitution } & Date & \multirow{5}{*}{ Pas de réel pic } & 15-mai-06 \\
\hline & Temps modal (j) & & entre 70 et $100 \mathrm{j}$ \\
\hline & Vitesse modale $(\mathrm{m} / \mathrm{j})$ & & entre 39 et 56 \\
\hline & Vitesse modale $(\mathrm{m} / \mathrm{h})$ & & entre 1,6 et 2,3 \\
\hline & $\mathrm{C} \max (\mathrm{ppb})$ & & 2,3 \\
\hline \multirow{2}{*}{$\begin{array}{l}\text { Moyenne (centre de } \\
\text { gravité du pic) }\end{array}$} & Temps (j) & \multirow{4}{*}{$\begin{array}{c}\text { Non calculable en } \\
\text { l'absence de données de } \\
\text { débit }\end{array}$} & 60,9 \\
\hline & Vitesse $(\mathrm{m} / \mathrm{j})$ & & 64,1 \\
\hline \multirow{2}{*}{$\begin{array}{l}\text { Bilan de la restitution } \\
\quad \text { (au } 23 \text { juin 2006) }\end{array}$} & Masse récupérée $(\mathrm{kg})$ & & 0,33 \\
\hline & $\%$ de la masse injectée & & $17 \%$ \\
\hline
\end{tabular}

Tableau 4 : Tableau récapitulatif de la restitution d'uranine au Puits Poubelle F2 et à la Fontaine 


\section{III.5 Traçage 2 depuis l'aven de Font Chapelle}

La sulforhodamine $\mathrm{G}$ injectée à l'aven de Font Chapelle a été détectée au Puits Poubelle F2 et à la Fontaine de Nîmes. Etonnamment, aucune trace n'a été détectée au Puits des 9 Arcades sur le bras Nord du réseau karstique, pourtant situé entre le point d'injection et la Fontaine. Les courbes de restitution de sulforhodamine G au Puits Poubelle F2 et à la Fontaine sont représentées à la Figure 4. La courbe de restitution à la Fontaine est de très bonne qualité avec un taux de restitution de $45 \%$. La restitution au Puits Poubelle est moins bien structurée. Il est par ailleurs surprenant d'observer des teneurs moindres au point de restitution amont F2 par rapport à l'exutoire aval. Deux hypothèses peuvent être avancées pour expliquer ce phénomène :

- Un autre cheminement du traceur qui serait réapparu via un autre chemin entre le Puits Poubelle F2 et la Fontaine y compris par le conduit Nord en aval du Puits des 9 Arcades;

- Un manque de représentativité du Puits Poubelle F2 qui serait situé sur un bras annexe du conduit Ouest alimenté par des venues latérales contribuant à diluer le traceur qui transite réellement au sein de ce conduit.

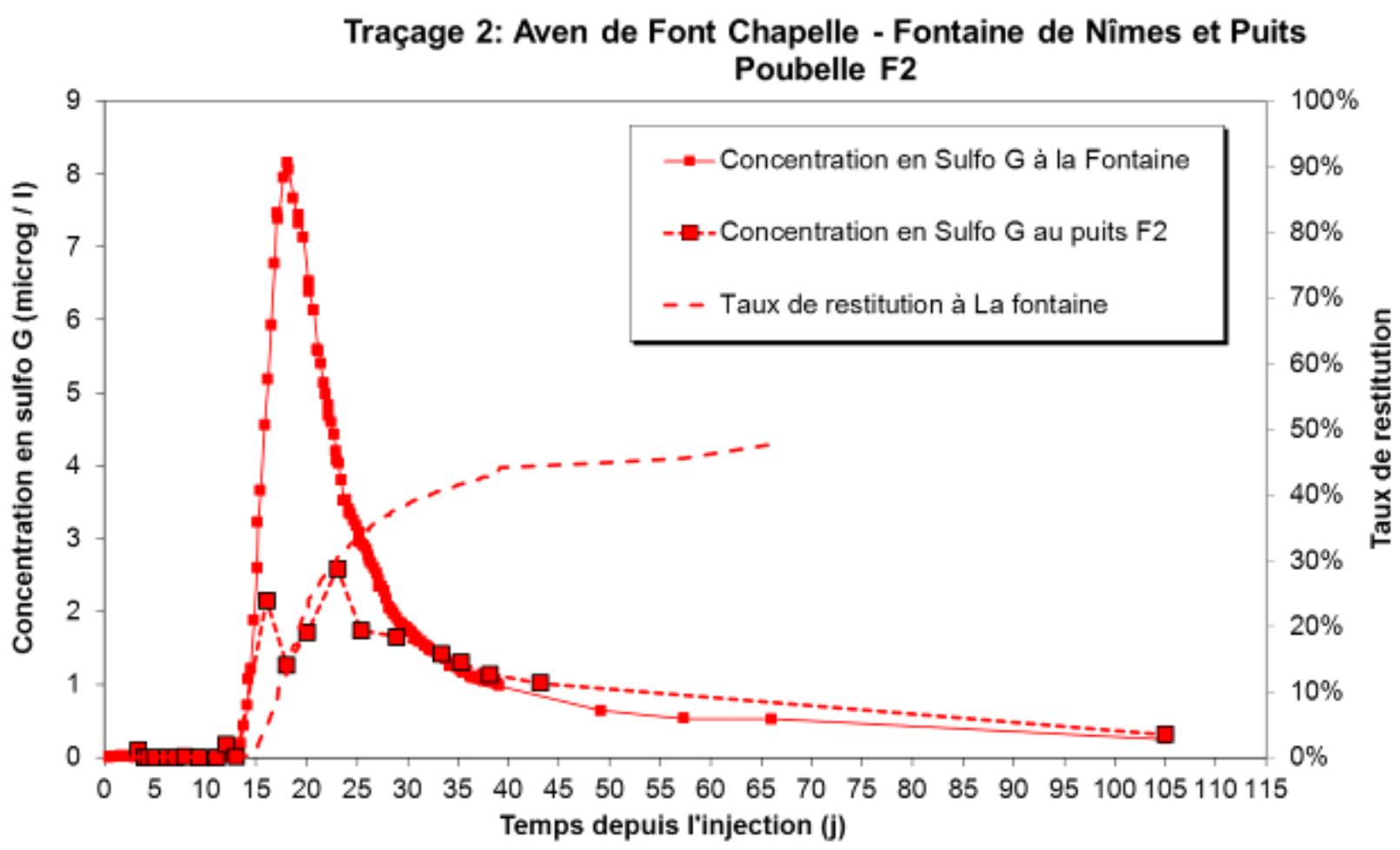

Figure 4 : courbes de restitution de sulforhodamine $G$ au puits Poubelle et à la Fontaine

Figure 4: sulforhodamin $G$ dye breakthrough curves at Poubelle well and Fontaine de Nimes

\begin{tabular}{|c|c|c|c|}
\hline \multicolumn{2}{|c|}{ Restitution des $3 \mathrm{~kg}$ de sulforhodamine $G$} & Puits Poubelle & Fontaine de Nîmes \\
\hline & Distance (m) & 2020 & 2250 \\
\hline \multirow{3}{*}{$\begin{array}{c}\text { Première arrivée du } \\
\text { traceur }\end{array}$} & Date & $20 / 03 / 2006$ & $23 / 03 / 2006$ \\
\hline & $\begin{array}{l}\text { Temps de transit } \\
\text { minimum }(j)\end{array}$ & 10,2 & 12,8 \\
\hline & $\begin{array}{l}\text { Vitesse maximale de } \\
\text { transit }(\mathrm{m} / \mathrm{j})\end{array}$ & 220 & 176 \\
\hline
\end{tabular}




\begin{tabular}{|c|c|c|c|}
\hline & $\begin{array}{l}\text { Vitesse maximale de } \\
\text { transit }(\mathrm{m} / \mathrm{h})\end{array}$ & 9,2 & 7,3 \\
\hline \multirow{5}{*}{ Mode de la restitution } & Date & $02 / 04 / 2006$ & $28 / 03 / 2006$ \\
\hline & Temps modal (j) & 23,1 & 18,1 \\
\hline & Vitesse modale $(\mathrm{m} / \mathrm{j})$ & 98 & 124 \\
\hline & Vitesse modale $(\mathrm{m} / \mathrm{h})$ & 4,1 & 5,2 \\
\hline & $\mathrm{C} \max (\mathrm{ppb})$ & 2,6 & 8,2 \\
\hline \multirow{2}{*}{$\begin{array}{l}\text { Moyenne (centre de } \\
\text { gravité du pic) }\end{array}$} & Temps (j) & \multirow{4}{*}{$\begin{array}{l}\text { Non calculable en } \\
\text { l'absence de données de } \\
\text { débit }\end{array}$} & 25,0 \\
\hline & Vitesse $(\mathrm{m} / \mathrm{j})$ & & 90,1 \\
\hline \multirow{2}{*}{$\begin{array}{l}\text { Bilan de la restitution } \\
\quad \text { (au } 15 \text { mai 2006) }\end{array}$} & Masse récupérée (kg) & & 1,37 \\
\hline & $\%$ de la masse injectée & & $45 \%$ \\
\hline
\end{tabular}

Tableau 5: Tableau récapitulatif de la restitution de sulforhodamine G au Puits Poubelle et à la Fontaine de Nîmes Table 5: recovery characteristics of sulforhodamine $G$ dye at Poubelle well and Fontaine de Nimes

Les vitesses des premières arrivées sont relativement élevées, comprises entre 7,3 m/h à la Fontaine et 9,2 $\mathrm{m} / \mathrm{h}$ au Puits Poubelle.

\section{III.6 Traçage 3 depuis l'aven du Pont de la République}

Les courbes de concentration de naphtionate au puits Poubelle et à la Fontaine de Nîmes sont présentées sur la Figure 5. La restitution du naphtionate de sodium à la Fontaine de Nîmes est bien marquée, avec des premières traces qui apparaissent le 17 mars, 7 jours après l'injection, soit une vitesse de $15,4 \mathrm{~m} / \mathrm{h}$, et un maximum de $144 \mu \mathrm{g} / \mathrm{l}$ au 19 mars, 9 jours après l'injection, soit avec une vitesse de $12,1 \mathrm{~m} / \mathrm{h}$ (Tableau 6). Le temps moyen d'arrivée correspondant au passage du centre de gravité de la restitution, est de 16 jours, soit près d'une semaine après l'arrivée du maximum du pic, ce qui correspond à une vitesse moyenne de $169 \mathrm{~m} / \mathrm{j}$ ou $7 \mathrm{~m} / \mathrm{h}$. Ces vitesses observées sont les plus élevées obtenues lors du multi-traçage. Vu le fort niveau de fluorescence «naturelle » présent dans les eaux de la Fontaine, il est difficile de déterminer la durée de la restitution, de même que ce bruit de fond peut entrainer des imprécisions dans la quantification des traceurs. $\mathrm{Au}$ vu de la forme de la courbe, on peut considérer qu'elle est de l'ordre de 10 à 12 jours. Au-delà du 26 mars, des traces de naphtionate semblaient cependant être encore mesurées, ce qui a notamment motivé la poursuite du suivi avec un pas de temps journalier jusqu'au 18 avril, puis de 8-10 jours jusqu'au 15 mai. Au passage du maximum de la restitution le 19 mars, la masse cumulée récupérée était de l'ordre de $3 \mathrm{~kg}$ (soit $24,7 \%$ de la masse injectée), et au temps moyen, le 26 mars, elle était de l'ordre de 7,3 $\mathrm{kg}$ (soit 58\% de la masse injectée). A la fin du suivi au pas de temps hebdomadaire, le 18 avril (soit 39 jours après l'injection), la masse cumulée récupérée est de l'ordre de $8,6 \mathrm{~kg}$, soit $69 \%$ de la masse injectée. Intégrer la courbe de restitution au-delà de cette limite ne présente guère de sens. En effet, vu le fort niveau de fluorescence «naturelle», il est difficile de déterminer si le signal est significatif ou non d'une présence de naphtionate. Le taux de restitution est néanmoins important, ce qui amène à penser que la connexion hydraulique entre l'aven du Pont de la République et la Fontaine de Nîmes s'effectue sans trop de «pertes », et que l'on est en présence d'un drain bien développé. La restitution au Puits Poubelle est bien moins structurée avec une première arrivée confondue avec le maximum du pic après environ $8 \mathrm{j}$. Ses caractéristiques sont décrites au Tableau 6. 


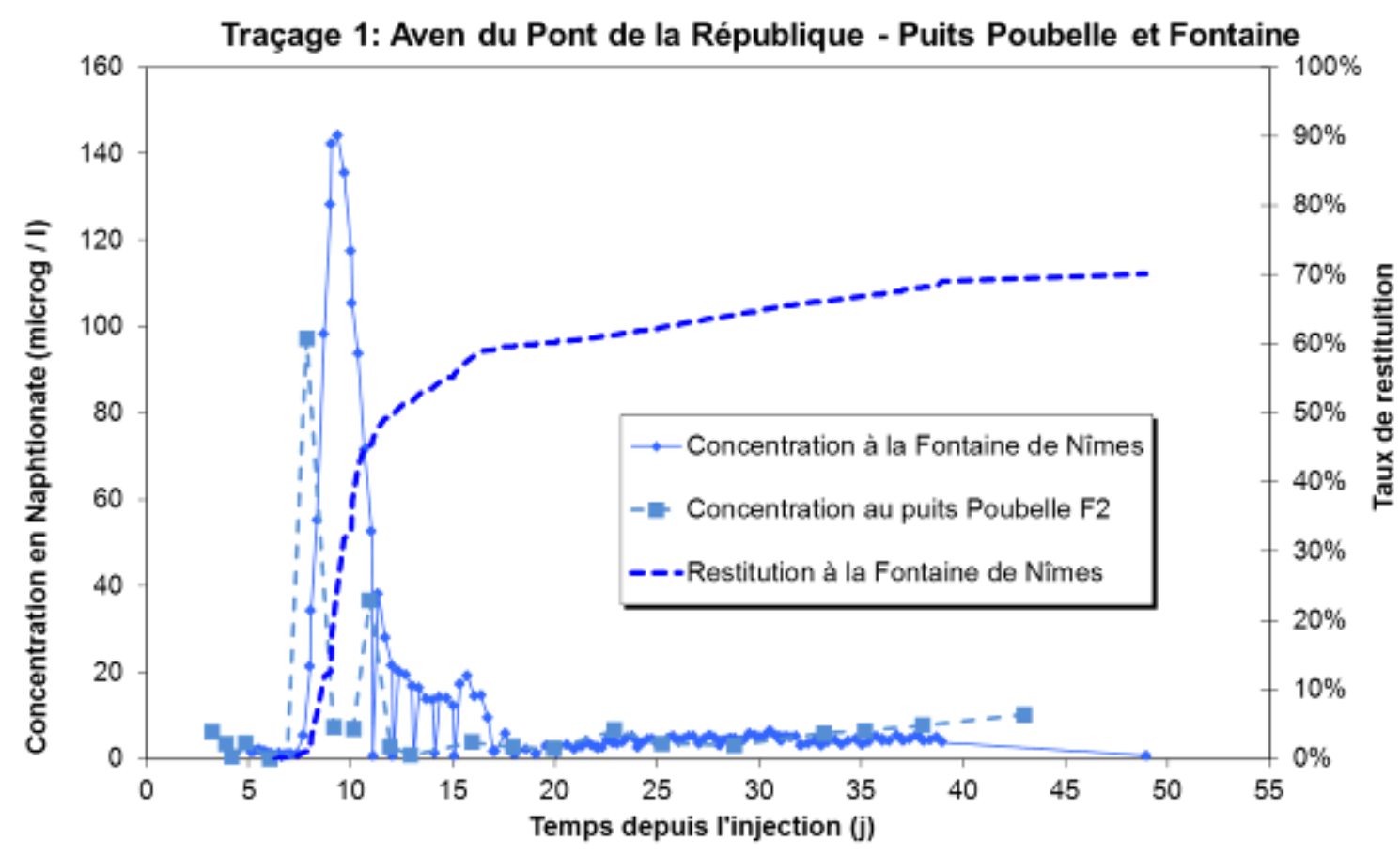

Figure 5 : courbes de restitution du naphtionate au puits Poubelle et à la Fontaine

Figure 5: naphtionat dye breakthrough curves at Poubelle well and Fontaine de Nimes

\begin{tabular}{|c|c|c|c|}
\hline \multicolumn{2}{|c|}{$\begin{array}{c}\text { Restitution des } 12,5 \mathrm{~kg} \\
\text { de naphtionate de sodium }\end{array}$} & Puits Poubelle & Fontaine de Nîmes \\
\hline & Distance $(\mathrm{m})$ & 2210 & 2710 \\
\hline \multirow{4}{*}{$\begin{array}{c}\text { Première arrivée du } \\
\text { traceur }\end{array}$} & Date & \multirow{4}{*}{$\begin{array}{l}\text { Quasi confondue avec le } \\
\text { maximum du pic ? }\end{array}$} & 17-mars-06 \\
\hline & $\begin{array}{l}\text { Temps de transit } \\
\text { minimum }(\mathrm{j})\end{array}$ & & 7,3 \\
\hline & $\begin{array}{c}\text { Vitesse } \\
\text { maximale de } \\
\text { transit }(\mathrm{m} / \mathrm{j})\end{array}$ & & 369 \\
\hline & $\begin{array}{c}\text { Vitesse } \\
\text { maximale de } \\
\text { transit }(\mathrm{m} / \mathrm{h})\end{array}$ & & 15,4 \\
\hline \multirow{5}{*}{ Mode de la restitution } & Date & 18-mars-06 & 19-mars-06 \\
\hline & Temps modal (j) & 7,8 & 9,3 \\
\hline & $\begin{array}{l}\text { Vitesse modale } \\
(\mathrm{m} / \mathrm{j})\end{array}$ & 282 & 290 \\
\hline & $\begin{array}{l}\text { Vitesse modale } \\
(\mathrm{m} / \mathrm{h})\end{array}$ & 11,8 & 12,1 \\
\hline & $\mathrm{C} \max (\mathrm{ppb})$ & 97 & 144 \\
\hline \multirow{2}{*}{$\begin{array}{l}\text { Moyenne (centre de } \\
\text { gravité du pic) }\end{array}$} & Temps (j) & \multirow{4}{*}{$\begin{array}{l}\text { Non calculable en l'absence } \\
\text { de données de débit }\end{array}$} & 16.0 \\
\hline & Vitesse $(\mathrm{m} / \mathrm{j})$ & & 169 \\
\hline \multirow{2}{*}{$\begin{array}{l}\text { Bilan de la restitution } \\
\text { (au } 18 \text { avril 2006) }\end{array}$} & $\begin{array}{l}\text { Masse récupérée } \\
(\mathrm{kg})\end{array}$ & & 8,6 \\
\hline & $\begin{array}{l}\text { \% de la masse } \\
\text { injectée }\end{array}$ & & $69 \%$ \\
\hline
\end{tabular}

Tableau 6: Tableau récapitulatif de la restitution du naphtionate de sodium au Puits Poubelle F2 et à la Fontaine de Nîmes

Table 6: recovery characteristics of sodium naphtionat dye at Poubelle well and Fontaine de Nimes 


\section{III.7 Traçage 4 depuis le Puits de la Gaffone}

La connexion étant fortement probable entre le Puits de la Gaffone et le Puits du Creux de Mouleri, plutôt que de traçage, il convient de parler d'un jaugeage du drain de la Galerie des Poteries effectué entre le point d'injection et le point de restitution.

Le principe général consiste à injecter une solution concentrée d'un traceur, et à rechercher dans quelle proportion cette solution a été diluée en aval du point d'injection. Cette dilution est fonction du débit, supposé constant le long du tronçon compris entre les deux points. La méthode consiste à injecter « instantanément» une masse connue de traceur $M$. Au terme d'un parcours suffisamment long pour que le mélange soit correct (c'est-à-dire que la concentration soit uniforme sur toute la section), la concentration $C(t)$ est mesurée pendant toute la durée $T$ du passage du traceur. En prenant l'hypothèse de la conservation de la masse de traceur, le débit moyen $Q$ peut alors s'exprimer sous la forme suivante :

$$
Q=\frac{M}{\int_{0}^{T} C(t) \cdot d t}
$$

avec $Q$, débit moyen pendant la durée de la restitution $T ; M$, masse de traceur injecté ; $C(t)$, concentration mesurée au temps $t ; T$, durée de la restitution en traceur.

La concentration en traceur est mesurée indirectement, en suivant l'évolution de la conductivité, qui est globalement proportionnelle à la salinité. L'équation (Eq. 1) admet implicitement que le taux de restitution du traçage est de $100 \%$, c'est-à-dire que la totalité du sel injecté au Puits de la Gaffone est récupéré au Puits du Creux de Mouleri. La courbe de restitution observée est présentée à la Figure 6. Les principaux résultats du traçage sont donnés au Tableau 7.

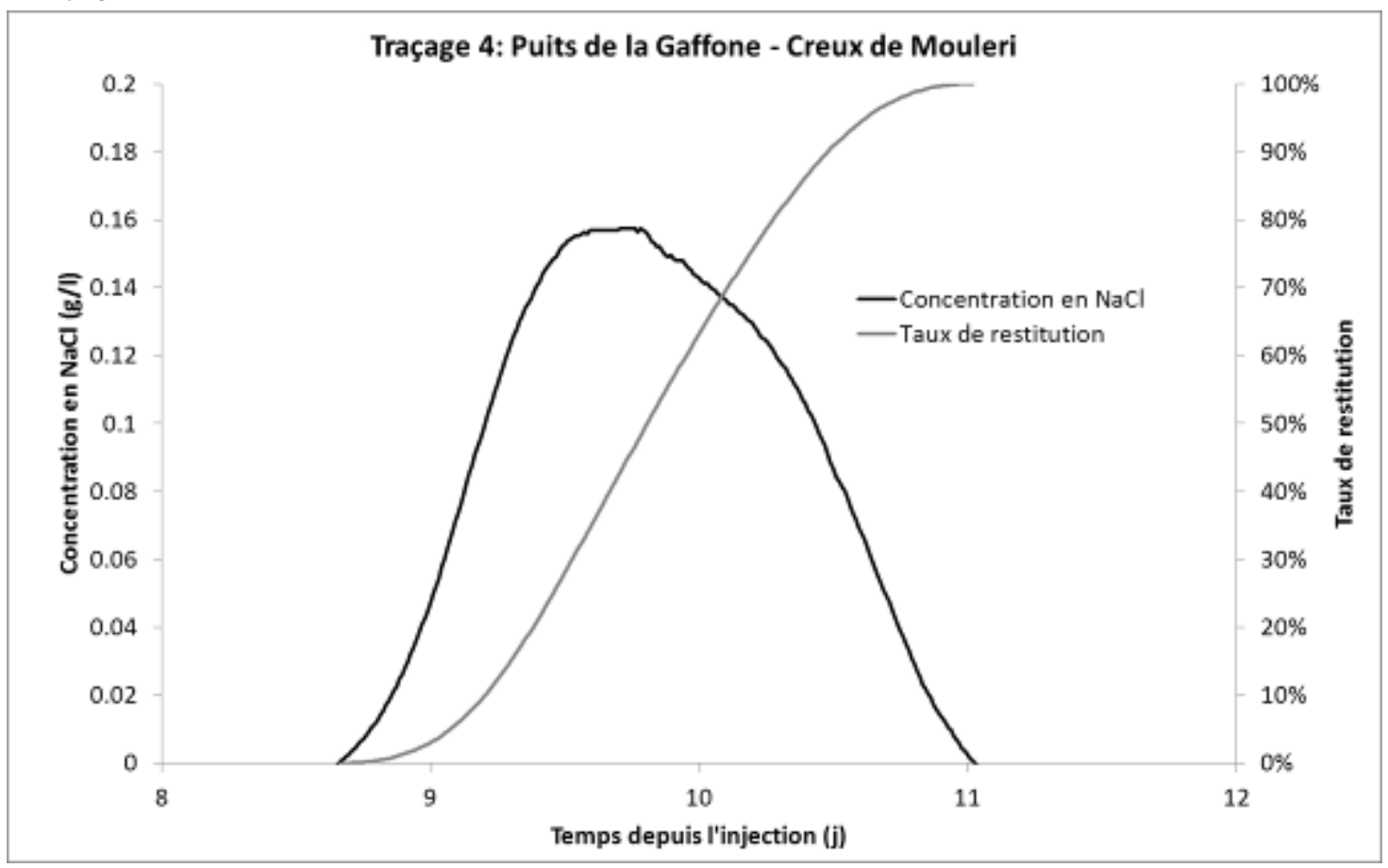

Figure 6 : courbe de restitution du NaCl ajouté au Puits du Creux de Mouleri Figure 6: salt breakthrough curve at Creux de Mouleri well

\begin{tabular}{|c|c|c|}
\hline \multicolumn{2}{|c|}{ Restitution des 15 kg de sel } & $\begin{array}{c}\text { Creux de Mouleri } \\
(\text { PCM })\end{array}$ \\
\cline { 2 - 3 } & Distance $(\mathrm{m})$ & 230 \\
\hline \multirow{4}{*}{\begin{tabular}{c} 
Première arrivée du traceur \\
\cline { 2 - 3 }
\end{tabular}} & $\begin{array}{c}\text { Temps de transit } \\
\text { minimum }(\mathrm{j})\end{array}$ & $19 / 03 / 2006$ \\
\cline { 2 - 3 } & $\begin{array}{c}\text { Vitesse maximale de } \\
\text { transit }(\mathrm{m} / \mathrm{j})\end{array}$ & 8,7 \\
\hline \multicolumn{2}{|c|}{} \\
\hline
\end{tabular}




\begin{tabular}{|c|c|c|}
\hline \multirow{4}{*}{ Mode de la restitution } & $\begin{array}{c}\text { Vitesse maximale de } \\
\text { transit }(\mathrm{m} / \mathrm{h})\end{array}$ & 1,1 \\
\hline & Date & $20 / 03 / 2006$ \\
\cline { 2 - 3 } & Temps modal $(\mathrm{j})$ & 9,7 \\
\cline { 2 - 3 } & Vitesse modale $(\mathrm{m} / \mathrm{j})$ & 23,6 \\
\cline { 2 - 3 } & Vitesse modale $(\mathrm{m} / \mathrm{h})$ & 0,99 \\
\cline { 2 - 3 } Moyenne & $\mathrm{C}$ max $(\mathrm{ppb})$ & 0,16 \\
\hline \multirow{3}{*}{ centre de gravité du pic) } & Temps $(\mathrm{j})$ & 9,9 \\
\cline { 2 - 3 } Conditions hydrologiques & Vitesse $(\mathrm{m} / \mathrm{j})$ & 23,2 \\
\hline \multirow{3}{*}{ Bilan de la restitution } & Débit $(1 / \mathrm{min})$ & 48 \\
\cline { 2 - 3 } & Masse récupérée $(\mathrm{kg})$ & 15 \\
\cline { 2 - 3 } & $\begin{array}{c}\text { \% de la masse injectée } \\
\text { (hypothèse du jeaugeage) }\end{array}$ & $100 \%$ \\
\hline
\end{tabular}

Tableau 7 : Tableau récapitulatif de la restitution du sel au Puits du Creux de Mouleri

Table 7: recovery characteristics of salt at Creux de Mouleri well

Le débit estimé au sein de la Galerie des Poteries en appliquant l'équation (1) est de l'ordre de 50 1/min. C'est un débit maximal correspondant à une restitution complète du traceur au point de mesure. Dans l'hypothèse où une partie du sel aurait migré vers un autre exutoire, le débit de la Galerie serait alors encore inférieur. Au même moment, le débit de la Fontaine de Nîmes était de $0,23 \mathrm{~m}^{3} / \mathrm{s}$. Le conduit karstique de la Galerie des Poteries draine moins de 0,5\% du débit de la Fontaine en période de moyennes eaux. Son bassin d'alimentation est très localisé ; en proportion du bassin de la Fontaine, cela représente de l'ordre de $0,2 \mathrm{~km}^{2}$.

La restitution au Puits du Creux de Mouleri est particulièrement brève $(2,3 \mathrm{j})$ pour une arrivée tardive $-9,9$ $\mathrm{j}$ en moyenne - du traceur, avec des vitesses plutôt lentes $(1 \mathrm{~m} / \mathrm{h})$ pour un drain karstique, liées à un très faible gradient hydraulique dans ce drain à développement principalement horizontal. Aucune fluctuation de salinité n'est détectée à la Fontaine de Nîmes.

\section{SYNTHESE ET CONCLUSION}

Les résultats du multi-traçage de la Fontaine de Nîmes sont synthétisés à la Figure 7.

Les trois traceurs fluorescents injectés à une distance de l'exutoire comprise entre 2 et $4 \mathrm{~km}$ ont été détectés à la Fontaine de Nîmes avec des taux de restitution compris entre 17 et 69\%. Les courbes de restitution à la Fontaine sont cohérentes et complètes, sauf pour l'uranine qui est arrivée plus tardivement à un moment où le pas de temps d'échantillonnage était plus lâche. Les vitesses moyennes des traceurs sont comprises entre 65 et $170 \mathrm{~m} / \mathrm{j}$.

Concernant les conduits karstiques, aucun traceur n'a été détecté sur le conduit Nord au niveau du Puits des 9 Arcades. Ce résultat laisse supposer que le conduit Nord joue peu de rôle sur les écoulements souterrains provenant de l'Ouest et du Nord du bassin mais drainerait de préférence l'Est du bassin d'alimentation de la Fontaine. Tous les traceurs fluorescents ont été détectés de façon significative au Puits Poubelle F2. Cependant, les courbes de restitution sont moyennement cohérentes et suggèrent que le point F2 appartient à un drain secondaire par rapport au conduit Ouest. Ces résultats soulignent l'importance, pour le suivi d'un traceur au sein d'un conduit karstique, de trouver un point d'échantillonnage représentatif du conduit. L'absence de naphtionate de sodium au Puits du Creux de Mouleri confirme que celui-ci ne draine pas le Synclinal de Vacqueyrolles et possède un bassin d'alimentation d'extension limitée.

Aucun des traceurs fluorescents n'a été détecté à la Maison Carrée. Si une relation existe tout de même entre le système karstique et les formations sédimentaires de la Plaine, les faibles concentrations en traceurs mesurées à la Fontaine, ajoutées à la dilution au sein de l'aquifère quaternaire, rendent impossibles la détection du traceur.

Le jaugeage au sel de la Galerie des Poteries indique que celle-ci draine un débit faible, de moins de 50 1/min. Les faibles vitesses $(23 \mathrm{~m} / \mathrm{j})$ sont liées au faible gradient hydraulique entre le Puits de la Gaffone et le Creux de Mouleri. 


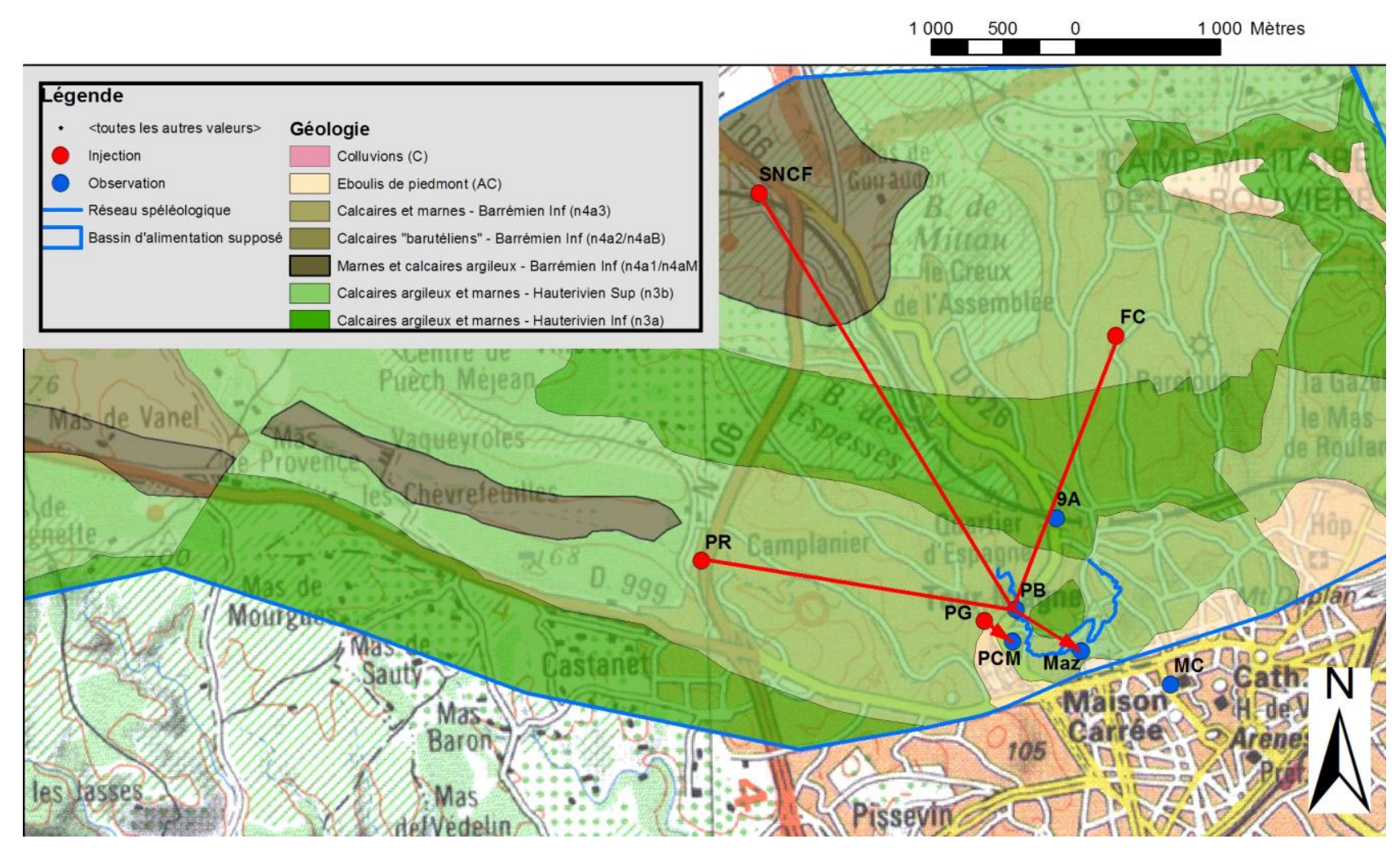

Figure 7 : synthèse des résultats du multi-traçage de mars 2006 (SNCF : Aven du pont SNCF ; PR : Aven du pont de la République ; PG : Puits de la Gaffone ; 9A : Puits des 9 Arcades ; PB : Puits Poubelle ; FC : Aven de Font Chapelle ; PCM : Puits du Creux de Mouleri ; Maz : Fontaine de Nîmes à l’Aven Mazauric ; MC : Maison Carrée)

Figure 7: results summary of the multi-tracing experiment of March 2006

\section{REMERCIEMENTS}

Cette recherche a été conduite grâce au financement de la Ville de Nîmes et du Ministère de l'Environnement et du Développement Durable. Les auteurs tiennent à remercier l'Association Fontaine de Nîmes (http://compo.free.fr/fontaine/), André Coste et Bernard Daudet pour leur collaboration.

\section{REFERENCES}

Coste A., Jouanen G. (2003) - Le «Creux de Mouléri »: un ancien regard sur le réseau souterrain de la Fontaine de Nîmes (Gard). Bul. Société d'Etude des Sciences Naturelles de Nîmes et du Gard, 2003, 64, p. 7-15.

Coste A., Jouanen G., Martin P., com. rédac. (1991) - Fontaine de Nîmes : Exploration spéléologique Nemausa XII - Collectif Asso. Spéléologique Nîmoise, Groupe Spéléologique "La Ratapenada », Spéléo-Club Lassallien Nîmes, pochette 16 p. + photos couleurs.

Coste A., Jouanen G., concepteur (2006) - Réseau souterrain de la Fontaine de Nîmes. Report en surface. Panneau informatif des Jardins de la Fontaine de Nîmes. Ed. Association Fontaine de Nîmes et Soc. Et. Sc. Nat. Nîmes et Gard.

Fabre G. (1997) - Le bassin d'alimentation de la fontaine de Nîmes d'après les expériences de traçages, Bul. Soc. et Sc. Nat. Nîmes et Gard, 61, p. 52-57.

Fabre G. et Guyot J.-L. (1988) - Sur l'hydrologie karstique de la Fontaine de Nîmes (Gard), Gard, Bul. Soc. et Sc. Nat. Nìmes et Gard, 58, p. 39-47.

Maréchal J.-C., Courtois N., Ladouche B. et Dörfliger N. (2006) - Jaugeage en continu de la Fontaine de Nîmes (Gard) : premiers résultats, Bul. Soc. Et. Sc. Nat. Nîmes et Gard, 65, p. 89-96. 
Maréchal J.-C., Ladouche B., Courtois N. et Dörfliger N. (2005) - Modèle conceptuel de la structure et du fonctionnement du système karstique de la Fontaine de Nîmes. BRGM/RP-53827-FR. BRGM. 187 p.

Maréchal J.-C. et Ladouche B. (2006) - Fonctionnement hydrogéologique du système karstique de la Fontaine de Nîmes en crue. Rapport final. BRGM/RP-54723-FR.

Maréchal, J.C., Ladouche, B. \& N. Dörfliger (2009) - Hydrogeological analysis of groundwater contribution to the 6-8 September 2005 flash flood in Nîmes, La Houille Blanche, 2, 2009, 88-93, doi:10.1051/lhb:2009019.

Maréchal J.-C., Ladouche B. et Dörfliger N. (2008) - Karst flash flooding in a Mediterranean karst, the example of Fontaine de Nîmes. Eng. Geol., 99, p. 138-146.

Nejad Hachemi, M.A. (1968) - Contribution à l'étude hydrogéologique de la région nîmoise.-, Faculté des Sciences. Institut de Géologie., Université de Montpellier, Montpellier. 